\section{Comparison of supplier quality assurance methods for compound feed manufacturing from efficiency, product consistency and economical point of view}

\author{
Andrea Csikai \\ University of Debrecen \\ Centre for Agricultural an Applied Economic Sciences, \\ Faculty of Agricultural and Food Sciences and Environmental \\ Management, Institute of Food Processing, Quality Assurance and \\ Microbiology, Debrecen \\ andrea.csikai@gmail.com
}

\section{SUMMARY}

Feed manufacturing and human food production are the main routes of use for agricultural products. The food industry particularly has intensively implemented the recent quality management principles and developed systems that facilitate the continuous improvement and efficiency of the industrial production. Feed production has taken similar approaches however the intensity of deployment at the manufacturer and the rollout towards its supply chain has shown slower progress. The methods, that the feed manufacturer manages the supplier chain of mainly primer agricultural products according to, have a certain impact on the efficiency of the inbound operations, feed product quality and its consistency as well as on other resources. These methods have been built on sound quality management principles that are stated not only in quality standards but also in relevant regulations. Current study addresses the questions related to the link between supply chain quality management and feed product. The objective of the frontier research was to highlight the theoretical possibilities and benefits of the robust design method implemented into animal feed manufacturing dealing with highly variable ingredients.

Keywords: supplier quality management, compound feed, formulation, robust design

\section{INTRODUCTION}

The various raw materials are the building blocks of feed products. Their quality has a direct impact on the quality of the final goods, even though certain manufacturing processes drastically convert the raw material, by changing the original attributes of the ingredient to product characteristics. Hence the quality control of the ingredients and the quality management of the supplier base are in the focus of every manufacturing operation. In the past decades activities and approaches to supply chain management changed due to the influence of market orientation. Secondly not only the approach itself went through changes, but also its magnitude was extended from vendors to the entire supply chain. The ultimate objective of the quality management is to maximise customer satisfaction with delivering high quality products and services for the value. Today, consumers look for a broad spectrum of attributes in their food purchase, therefore the consumer is the one, who essentially determines what food products should provide. Similarly to food, feed product quality attributes are to be defined more and more by the end users too. However, on one hand, the "end-user" is the target animal group eating the feed. On the other hand, the owner or farmer is also the end-user, who uses the feed product and lastly pays for it. Consequently, a precisely and accurately engineered feed product must provide optimal solutions in terms of both aspects of use.

It is worth to emphasize that the aim of product design empowered by quality management is to provide the same value for money, consistently, all the times, through every purchase unit of the product. Therefore, the robustness of the system is a key component to determine the success on market. The measurement of customer satisfaction is the elementary indicator of a product success that is, practically speaking, the foundation stone of the company's future.

Those suppliers, that cannot manage to deliver products against expectations contracted according to specifications, generate on-costs to the manufacturer and may significantly impact market success of the product. These failures and the losses caused by are not affordable in today's environment. On the contrary, these suppliers are impacted indirectly as well, due to the possibility, that the producer may choose different vendors or unable to maintain the operational relationship through ordering further quantities from the vendor. In the recent times, the aim of quality management principles went beyond the boundaries of a single company and the entire product chain got into the spotlight. Although breaking down the barriers and rolling out stringent processes, in practice, it seemed to be a rather challenging exercise on industrial scale. Furthermore, even though the customer expectations became relatively high towards the products and the participants of the food chains, agricultural operations, providing raw materials for human food and animal feed processing, had a delay to follow the tendencies spread on the markets.

By the definition of the market orientation concept, products are to be designed according to consumer needs and requirements. According to the conclusions drawn by Grunert et al. (1996) based on their study, agricultural and food companies need to develop more understanding of their markets and apply the knowledge to gain competitive advantage through the application of market-orientation approach. 
A brilliant example can be taken from the car industry to back up the theory, that there is a gap in customer understanding and the transformation of needs into product concept. It has been brightly recognised by Henry Ford, when the first automobile was designed, customers would have asked simply for a faster horse.

Meyer and Schwager (2007) have taken cognizance of the role of product development, which is not only specifying the product attributes, but also designing experiences based on observations about how customers use products, generating learning why they use offerings, as they do. These activities trigger further insights for product development towards new or modified product features.

In terms of food and feed production, the manufacturers deal with primer agricultural products. When product designs are ameliorated, the product attributes are translated to ingredient specifications, via the identification and selection of raw materials. Nevertheless, misalignments eventuate during this translation flow. Nonetheless innovation in the feed sector accelerates, the reaction of agricultural production to the changes of customer expectations is relatively slow compared to other industries.

Ondersteijn et al. (2006) found that many performance indicators are available at company level, however there are three key obstacles to maximise performance of the chain. Partners do not always have the same objectives, in line with the optimal performance of the total chain. Furthermore, the relevance of information is different on each level, even if it is of high importance for the overall chain. Thirdly, the strategic value of the information inhibits a free exchange between the partners. Integrated supply chains offer opportunities for creating further added values that the single firms could not achieve alone (e.g. labelling or traceability systems). The specific issues of agri-food chains are largely determined by inherent characteristics of agricultural production. The most important ones are the biological character of agricultural production, its close relation to and dependence on nature, and the perishable nature of the products.

Boehlje and Schrader (1996) have recognised that the information, about how to produce required product attributes, has a fundamental position of power in the supply chain. The authors state that the value adding resources in differentiated product markets are more in the form of information, than hard assets. In the study two fundamental points of control and one fundamental source of power have been defined. The points of control in the chain may be at the beginning (consumer) and at the end (raw materials). The source of this control is the knowledge. At the consumption end, it is knowledge of the consumers' needs that can be communicated and/or dictated to the rest of the food chain. At the raw material supplier end it is knowledge and information about the genetic material or the technology that can produce at a lower cost, in a shorter time those specific attributes which consumers are willing to pay for. As the source of control is knowledge and information (not physical resources e.g. capital, land), then the only way, a firm can obtain control between the end points of the consumer and the genetics company, is through superior information. For that very reason, agricultural companies providing raw materials for the feed manufacturing play a fundamental role in ensuring safety quality of products.

The product development and quality management are the power engines of companies to generate knowledge and information about their own products and the links to the down- and upstream steps of the supply chain. Firms cannot improve things that they do not evaluate, and they also cannot evaluate them without right measurement. Companies established sound performance measurement methods and tools in order to optimize internal production processes, to attain targeted product quality and conformity and to improve the efficiency.

In Shewhart's (1980) definition, quality can be described as numerical measurement that makes possible to see if the quality of the product for a given period differs from that for some other period taken, as a basis of comparison. It can be also the comparison of qualities of product for two or more periods to determine if the differences are greater than should be left to chance. He has addressed that from industrial quality control point of view the establishment of standards is necessary and they should be expressed as quantitatively measurable physical properties. In his view, the role of an engineer (nowadays, a manufacturer firm) is to try satisfying consumer needs by translating them, as nearly as possible, into physical characteristics of the product. Additionally, the role also includes setting up ways and means of obtaining a product that does not differ from the standards more than may be left to chance.

Deming (2000) underlined the importance of standards and measurement in buyer and seller relationships. The specification of an item is meaningless without operational definition. It must contain quantitative attributes and refer to the measurement considering that the instruments are in statistical control. The principles, laid down by Shewhart and Deming, provide the universal foundation of modern quality management. The iterative steps of Plan - Do - Check - Act are the milestones on the way of continuous improvement and they are applicable to every operation steps, including supplier quality management too.

The ISO 9001:2008 quality management standard incorporates the continuous improvement approach to achieve customer satisfaction, through consistent products with the active contribution of each operative function of the organisation. The standard has specific requirements for the control over purchased products. In the purchasing relevant section it states that the company should ensure that purchased product conforms to buying requirements. Typically, for this purpose, raw material 
specifications are commonly used as part of the contractual agreement between the vendor and buyer. The standard puts the type and extent of control, applied to the vendor and purchased material, in the context of the effect of the product on the subsequent processes or the final product. Logically, in line with Deming's theory, the evaluation and selection of suppliers should be based on their ability to provide ingredients in accordance with the specification. Inspection systems or other necessary activities need to be implemented to verify that bought products meet the specified requirements. In food and feed industries inbound quality checks act the part of inspection, coupled with systems establishing more forward control at vendor level (e.g. supplier auditing). The forward controls may highly contribute to the efficiency of inspection systems and highlights additional areas that potentially could cause quality incidents at the buyer.

Mittag and Rinne (1993) distinguished three types of deviations. Namely these are the deviations within a unit, deviations between units and deviations over time caused by one of the four M's (man, machine, method, and material) or the environment. During the design process of a product, when quality attributes are defined, these influences must be considered with tolerance ranges around the nominal values. Quality management empowered by statistical tools is to ensure that the manufacturing process stays within this range. The data for the analysis is mainly derived from samples, but the results may trigger taking the action of screening. Certain quality characteristics are necessary to be screened, but some cannot be due to the nature of the inspection that destroys or reduces fitness of the material. The advantages of sampling over screening are the speed to obtain results, the lower cost, and that the sampling can be performed more thoroughly.

Gunasekaran et al. (2004) developed a framework to promote the importance of the performance measurement and metrics related to supply chains. In their survey six key performance indicators (KPI) were analyzed related to the supplier link. These were supplier delivery performance, lead-time against industry norm, supplier pricing against market, efficiency of purchase order cycle time, efficiency of cash-flow method, and supply booking procedures. Quality of delivered goods has been identified as the first in importance, followed by on time delivery and the flexibility of service systems in the delivery performance category. To ensure improvements, the control of processes in a supply chain is necessary through measurement and comparison to a set of standards with limits.

In the food industry strict supplier performance measures and processes have been introduced and combined in scorecards with weighted scores on various aspects of delivery against the contract terms. Scorecards provide transparency for supplier ranking and grouping to approved, approved for development, non-approved categories.

Jones (2000) provided practical advices about good ingredient quality control program for feedstuff producers. These companies use by-products of other industries in great amounts, however, at the same time, trying to manage minimal variation of finished feeds. The nutrient variations of these ingredients violate the nutritional profile of the feed formula and costs in terms of performance. The analysis of proximate (moisture, protein, fat) parameters of feed ingredients by Near Infrared Spectrophotometry (NIRS) on real time basis provides certain information about the variance, although many manufacturers do not analyze ingredients prior to use. High quality ingredients are predictable and constant in quality. To measure raw material quality, ingredients need to be described in terms of analytical values (with verified accuracy), physical and/or sensory characteristics. Jones points out that the ingredient quality received may well reflect of what the suppliers believe the buyer wants in terms of quality, so the communication between the parties is crucial. Detailed specification is a critical element of the communication. Vendor's agreement needs to validate the conformity to the specification. Welldefined sampling procedures and the management of rejected deficient loads also provide information to carry out evaluations of the supplier's performance that should be shared with the vendor on a regular basis. Formula adjustments to the actual assays have a high importance as it balances the product design into the acceptable ranges.

According to the Taguchi method (robust design) the control and elimination of variations, in terms of quality, could happen in the design and production of an item through system, parameter and tolerance design. In the first stage the system is designed and generated as a concept, idea or method. In the parameter design stage nominal values are determined for each quality parameter. It improves the uniformity of the product without cost or even with saving. In other words, certain parameters of a product or a process could be defined to make the performance less sensitive to variations. The third stage, tolerance design, improves the quality at a minimal cost by understanding the effect of parameters and by tightening their tolerances to reduce variations. This step also frees up resources so that their allocation can be optimised. The Taguchi method does not consider quality of a product if it is in- or outside of the specification limits, however it does, as the variation from the target that consists of real variability and bias. The application of the robust design principles reduces product variation through the product design that is less affected by variations. The tolerance design step deals with the allowed level of variation (Ross, 1996).

\section{MATERIAL AND METHOD}

To assess how feed manufacturers can shape the supplier chain quality management system and what benefits and drawbacks the manufacturer may face when he chooses the procedure of quality control, a compound feed product development cycle have been simulated both with linear and stochastic 
programming. The all information packages, that are essential for formulation, have been listed and grouped according to the source. The information flow between functions, roles at the feed manufacturer and suppliers has been mapped with the intention to apply the Taguchi robust design method not only for internal but also extending it to supplier chains.

The central element of compound feed design, the translation of customer needs to product specification, is mainly the activity of ration formulation. A theoretical framework had been developed to holistically integrate the product, formulation and supply chains from design phase to real production. The overall objective of this frontier research was to achieve constant quality and food safety of the product, on the least cost, through effective supply chain and flexible formulation methodology. The sound information and the understanding derived from the system establish interaction between these two steps.

To identify potential factors, that may influence specific product attributes, Ishikawa diagrams were created, because each cause can generate quality defects. Due to the subject of this study, from the major categories (people, methods, materials, machine, measurement, environment) the spotlight has been directed onto raw material relevant causes to enable to have tailor-made vendor quality control program.

Formulation was based on actual raw material prices, the average and the standard deviation of ingredient parameters chosen, and set probability level to find the least cost solution within the specified constraints. The constraints were assumed to reflect the customer expectations from nutritional and functional aspects. In the design phase several iterations were made to design the conceptual product from raw material portfolio.

The design phase of the product included the definition of ingredient specifications based on capability analysis of suppliers in terms of delivery consistency on each quality and food safety parameter that the ingredient may influence significantly. Raw material quality attributes were grouped around food safety, nutritional, functional dimensions and risk assessments were done for every raw material source with the assessment of intrinsic risks. Parameters for the specification definition have been selected through risk assessments, considering the likelihood of occurrence and the severity of effect in the context of the impact on the final product. The result of capability analysis has been used down- (for specifications and supplier development) and upstream (to reformulate rations with actual standard deviations of the parameter/raw material combination from the suppliers in question).

Generalized decision tree was set up for ingredient supplier quality management with recommendations for control methods. If raw material potentially had a significant impact on final product quality or food safety, the vendor assurance program was designed to evaluate control measures in place at the vendor that efficiently manages the risk. The necessary steps of validation in the design phase, and verification as a timely swift towards the first production were also outlined. The main inbound control procedures that feed manufacturers use to prevent deficient material entering the production site have been SWOT (strength, weaknesses, opportunities, threats) analysed and used for comparison.

\section{RESULTS}

In the contemporary compound feed manufacturing ingredients from agricultural chains are used as materials by nature that the formulation software prefers to incorporate into the ration, as the best fit to the constraints on the least cost. The formulations are mainly achieved by linear programming that only considers one figure (typically the average) for a nutritional or other parameter populated in the software. Consequently, variability of the parameters is not considered or reflected in the rations. In order to balance the variation of ingredients and its impact on end-product consistency nutritionists often use safety margins in the calculations that drives ration costs up, and it does not reflects the actual raw material variability. If a manufacturer intends to take advantage of the underutilized possibilities in the supply chain and gain deep understanding of ingredients and variations, one may need to design products that provides consistent quality by managing not only internal processes but the variability rooted in the supply chain. Various quality management principles suggest applying measurement on supplier performance (e.g. scorecards). It helps not only the buyer to have objective basis for comparison of vendors but also the data shared with the vendor enables the overall improvement of the link in the supply chain. An additional opportunity rises, if the results of supplier performance assessment (data of inspections) are fed back to formulation. Figure 1 . displays the decision tree about the mapped information flow related to a compound feed design process and the links to supply chain quality assurance.

After the initial ration (draft recipes) of a new product has been generated, the company needs to find and establish link with vendors whose products (the raw materials for feed production) are capable to deliver the required quality parameters constantly. This stage may be iterated a few times, from the design and formulation stages to vendors and back, depending on availability, or quality of ingredients from various suppliers.

According to the robust design method these loops have primer importance of final design that considers variations, although, due to the length of the process, and pressure on speed-to-market, it does not fully happen in appropriate depth. It also means that opportunities are lost to reduce variance of the product that may cause economical losses by the performance of the target animal group. Of course, in 
addition, further variances may occur, driven by internal processes at the feed manufacturer that were out of the scope of this study. The sign-off of specifications should be the outcome of the validation of suppliers' capability to deliver ingredients according to the required quality. Statistical analysis of data from validation can provides transparency about the standard deviation and average of the parameter from that certain vendor. The knowledge of descriptive statistical results should be incorporated into the specifications (referring to the tolerance design concept) and helps to refine ingredient parameters used in formulation. This way the awareness is obtained in the design phase of a product, rather than in the operational stage, when raw material lots get rejected due to nonconformity.
Furthermore, the validation is followed by verification after the development stage. Verification proves that the specified limits are achievable and provides the material that was needed for the diet. The verification supported by supplier audits (to check vendor processes) and delivery inspections give assurance that the system built up will provide safety and consistency. However, it must be underlined that the frequency of auditing and inspection must be set in line with inherent risk of the ingredient and the factors influencing it at the supplier. Materials with higher risk threshold and suppliers, whom the confidence has not been built with yet, are checked and monitored more frequently. If the construction of forward control system is not possible for any reason, screening is the way to gain the same information that was not available from the supply chain.

Figure 1: Links between product development (formulation) and supplier quality assurance from the design to the production of a new feed product

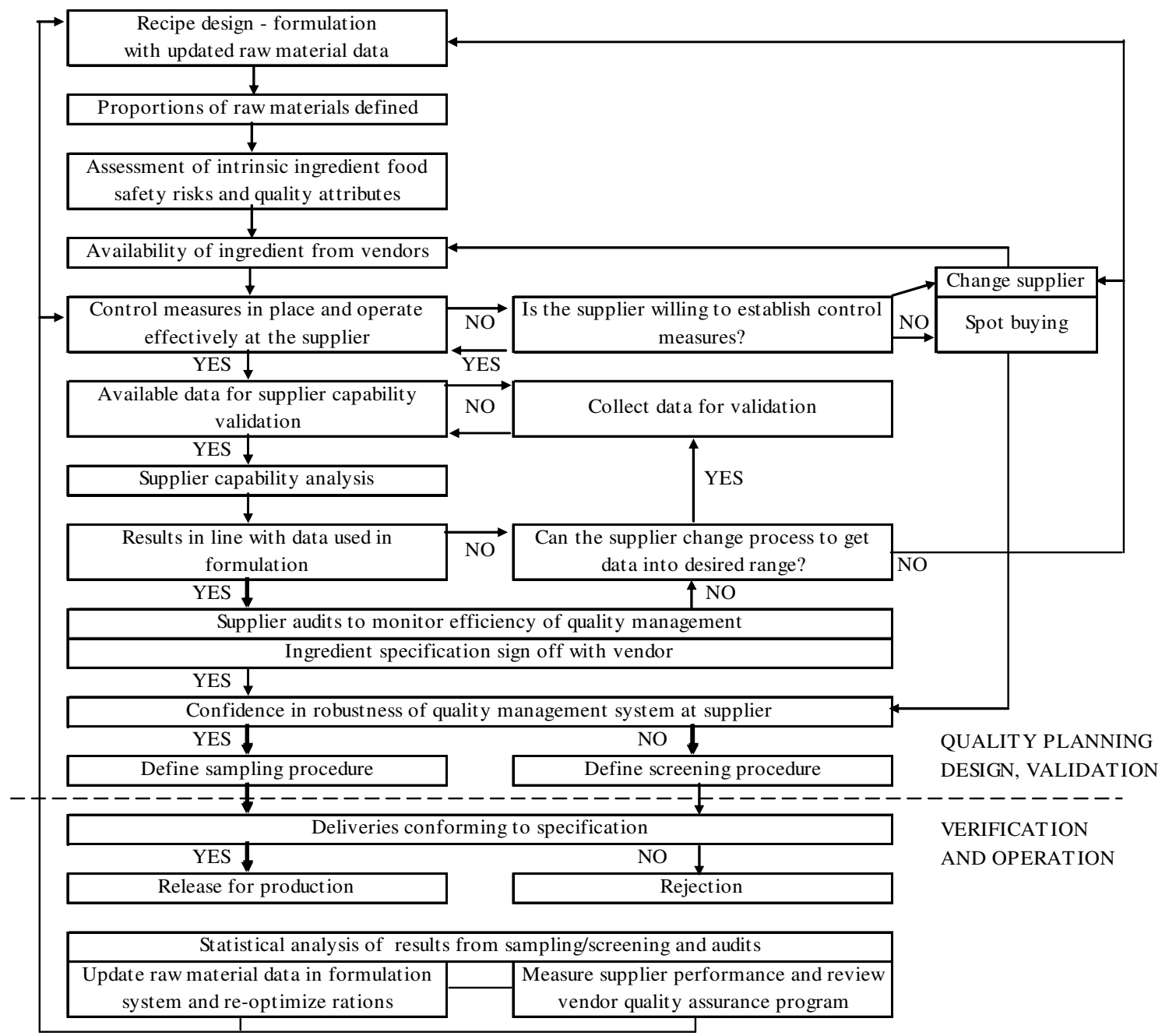

The operational success of both ways (sampling, screening) require quite significant resource allocation from feed manufacturer, including costs of analysis, manpower invested in carrying out audits, sampling and checking delivery batches, time to release lots for production. The SWOT analysis of the possible solutions to generate information about the supply chain has shown the forward control, as a combination of sampling and auditing, manages resources more efficiently. The resources are 
allocated to those steps where the risks occur and could be controlled, monitored, so the corrective actions can be determined in a timely manner. Less time is taken to carry out the limited number of inspections. Also, some data may come from the vendors that make the operation leaner. The frequent personal contact with the suppliers (via audits) can establish mutually beneficial relationship between vendor and buyer to enable strategic alliances for the future. It was identified as a main threat that if sampling frequency and methodology were not defined in a statistically correct way, it may distort the evaluation and hide problems.

The screening of every single delivery lot on the specified parameter generates sound data set reflecting the real variability of the material. It also enables that the buyer can react on real time on every deviation and make decision about the use of the material, so indirectly the product quality vary less. On the other hand the method is very resource intensive. As every batch needs inspection the speed to get the material into production reduces and the infrastructure should facilitate the increased need for storage room of batches on-hold. In special cases (e.g. buying via traders) there is no possibility to share results with the original producer of the ingredient. It is a great opportunity, that the data about the actual batches are available on real time basis. If the upstream processes support it, the manufacturer may re-formulate rations with actual figures, so that the variation on product level is eliminated by the formulation activity carried out in very high frequency. Although in this case, automatisms must be in place to ease the management of increased number of changes. From traceability point of view, a high number of alternative rations for the same design will be created, that needs appropriate management as well.

\section{CONCLUSIONS}

As said in the literature, the fundamental position of power in the supply chain is information. As presented above, the feed manufacturer has the opportunity to derive and aggregate superior data about the ingredients, suppliers, develop internal know-how to improve and strengthen the position on the market. However, for this purpose the measured data need to be collected, processed and actively used for development. Otherwise the unique opportunity is lost.

Agricultural products are varying (seasonality, geographical sources, etc) and they do so continuously and significantly. The specification, what applied a year before, might not be relevant any longer because the raw material has changed. Secondly, prices of raw materials and availabilities are also changing all the times, so a feed producers re-optimize rations frequently. So none of the elements of the initial design stay constant across the product lifecycle. A system developed, to make the prevention and reactions possible in case of the changes, therefore needs high level of flexibility and operational speed.

Considering Taguchi's robust design in the context of feed production, the relevance of the principles is even more obvious if the product design and formulation follows the stochastic approach. Stochastic formulation takes into account the variance of parameters and finds a safe diet proposition. On long term, the likelihood of that the product on the market is more consistently in line with customer expectation goes up. Following the same concept in the product design phase, not only the products, but also the supplier quality assurance system could be formed to minimise "noise" of quality deviations.

The market orientation in feed production is less a trend than in other segments of agriculture (e.g. horticultural vegetable production). Although the direction is likely to be the same, the rollout and deployment are likely to take longer in animal feed production, maybe because measures are also not fully in place at the place of use. There is a drift in thinking about agricultural products as conventional, "taken as they were given by nature" materials, towards ingredients "grown for special purpose". To achieve this, all partners along the production chain should get aligned view and cooperate.

\section{REFERENCES}

Boehlje, M. D.-Schrader, L. F. (1996): Agriculture in the $21^{\text {st }}$ century, Journal of Production Agriculture, 9. (3.) 335-340.

Deming, W. E. (2000): Out of the crisis. MIT Press. 167-182.

Grunert, K. G.-Baadsgaard, A.-Larsen, H. H.-Madsen, T. K. (1996): Market orientation in food and agriculture. Kluwer Academic Publishers. 3-18

Gunasekaran, A.-Patel, C.-McGaughey, R. E. (2004): A framework for supply chain performance measurement. International Journal of Production Economics, 87. 333-347.

Jones, F. T. (2000): Quality control in feed manufacturing. Feedingstuffs, 72. (29) 85-89.

Meyer, C.-Schwager, A. (2007): Understanding Customer Experience, Harvard Business Review, (2) 1-12.
Mittag, H. J.-Rinne, H. (1993): Statistical methods of quality assurance, Chapman \& Hall, 7-13

Ondersteijn, C. J. M.-Wijnands, J. H. M.-Huirne, R. B. M.Kooten, O. V. (2006): Quantifying the agri-food supply chain. Springer, Netherlands, 3-38.

Ross, P. J. (1996): Taguchi techniques for quality engineering: loss function, orthogonal experiments, parameter and tolerance design. McGraw-Hill Professional, 203-245.

Shewhart, W. A. (1980): Economic control of quality of manufactured product. American Society for Quality. 37-54.

ISO 9001:2008 Quality management systems - Requirements 